\title{
Enhancing the motorcycling experience with social applications: A study of user needs.
}

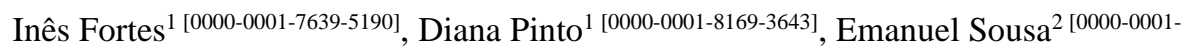 \\ 5128-5753], Vera Vilas-Boas 3 [0000-0003-3509-074X], and Rui José1 [0000-0003-3547-2131] \\ ${ }^{1}$ Algoritmi Research Centre, University of Minho, Portugal \\ \{ines.fortes, dianapinto\}@algoritmi.uminho.pt, rui@dsi.uminho.pt \\ ${ }^{2}$ Centro de Computação Gráfica, Guimarães, Portugal \\ Emanuel.Sousa@ccg.pt \\ ${ }^{3}$ Bosch Car Multimedia, Braga, Portugal \\ Vera.VilasBoas@pt.bosch.com
}

\begin{abstract}
For many riders, the motorcycle is much more than a transportation mode. Riding a motorcycle can be a pleasurable experience in itself, and the motorcycle is frequently a tool of socialization. An evidence for that can be found in the numerous motorcycling communities around the world. However, those communities may not be accessible to everyone, or they may not satisfy everyone. We believe that a social application for motorcyclists could reach more riders and has the potential of more easily fitting their needs. To explore this idea, we conducted focus groups with motorcyclists, exploring their current practices in digital tools as well as their needs and desires that could possibly be integrated in a social application. The results hinted on several aspects that need to be considered in the design of a social application for motorcyclists. Generally, motorcyclists are willing to receive and share information with others, and referred several needs in terms of communication and trip planning. However, they also showed concerns regarding a digital tool associated with riding, both for safety reasons and because it could disrupt the riding experience. We conclude that not only the needs but also the concerns must be considered in the design of this new digital tool.
\end{abstract}

Keywords: Social Application, Motorcycling, User Research.

\section{$1 \quad$ Introduction}

Riding a motorcycle can be much more than traveling from A to B. For many riders it means adherence to a set of cultural and social practices that distinguishes a particular and identifiable way of living. It is common to find motorcyclists who grew up around motorcycles and soon developed a fascination for these vehicles. Others may only embrace this world later in their lives, but they end up making it an integral part of their lifestyle. For many of them, riding a motorcycle almost defines them as a person, giving them the feeling of being part of a community of riders and a sense of belonging that only members can fully understand and share [1]. In fact, for many riders this social 
component is a defining element of being a rider [2, 3]. However, buying a motorcycle does not buy a social experience, and many riders may never really find the right context to experience that sense of belonging and companionship that others often praise as one of the main reasons why they love being motorcyclists [1].

One might place riders in a continuum of social engagement: at one extreme there are those (social riders) who mainly ride for leisure and like to ride in groups and share their passion for riding; at the other extreme, there are those for whom a motorcycle is mainly a utilitarian vehicle for commuting (commuting rider), and for whom the social experience is not seen as very important. Despite these differences, both these profiles, as well as the many others in between these two extremes, could benefit from some type of social applications that could enhance and bring new opportunities to the riding experience.

For the social riders, it should be easy to find other people with similar interests, so that their experience could be mutually improved. These riders may buy a motorcycle in the expectation of a certain social experience, but they may lack a group to share their experiences with. They they may also find it difficult to engage with the many others who share their passion for motorcycles, but who are outside their immediate social circle. These riders could benefit from social tools that give them the flexibility to promote and find new riding experiences and engage with broader and less rigid concepts of group.

For the commuting riders, engaging with other fellow motorcycle commuters could offer opportunities for the collaborative improvement of riding comfort and safety. Instead of being just by themselves, being a member of a broad community of riders could increase awareness about their daily difficulties and give them more power to promote change. For instance, they could promote campaigns so that other (e.g., four-wheel) drivers could learn more about how to drive near motorcyclists, and motorcyclists could use collective intelligence to increase awareness about their effective driving capabilities and improve their riding skills.

Digital technologies can be key enablers for many new forms of social engagement between motorcyclists. However, so far, the digital element of that experience has been limited to the before and the after moments of riding itself. Social applications used by motorcyclists are essentially generic services that allow users to share content related to their events and their shared passion for motorcycles. To improve the motorcyclists' experience, we should also consider how engagement between riders might occur, in the context of a continuous social experience that can span all the way from the cosy environment of the living room (when a ride is still just a plan for the upcoming weekend), to the most thrilling moments of riding along with other motorcyclists. However, the level of concentration and physical control needed to safely ride a motorbike represent huge hampering factors for common forms of digital interaction [4]. For obvious safety reasons, most interaction possibilities are either too limited, too intrusive or simply not acceptable when riding, making them technological hindrances to the riding experience. Moreover, even when adequate solutions may exist, an unequal access to them (e.g., due to price) has a negative impact on social collaborations while riding.

In this research, we aim to understand the needs of motorcyclists regarding digital features that could connect them, in any possible way, with other motorcyclists and the 
broader motorcycling ecosystem. Our goal is to inform the design of a new generation of social applications conceived specifically for the motorcycling experience. These social applications could significantly enhance the social experience of many riders, while hopefully helping to create services that benefit the whole motorcycling community.

\section{Related work}

Recent years have witnessed a strong growth in communication, navigation and entertainment systems for vehicles, including motorcycles [5]. Current research is addressing almost any element of the riding experience, including, for example, safety technology based on the interaction of vehicle-to-driver/environment [6], communication between motorcyclists [7], and communication between motorcyclists and other vehicles to signal the presence of each other to prevent accidents [7]. Additionally, studies are also focusing on vehicle-to-vehicle or vehicle-to-infrastructure communication, to share information related to routes, weather, traffic and restaurants [8]. Importantly, there have also been efforts to develop technology for promoting social interaction between motorcyclists $[4,9]$.

Some motorcycle communities promote all sorts of social interaction, for purely recreational purposes or more formal goals such as fundraising, competitions, political protests or community services [10]. However, it is important to note that online communities may differ from the physical ones. For instance, when bikers engage in online communities, they tend to communicate more with photos (e.g., of their vehicle) or videos, rather than with written text [11]. However, online and other communities also share communalities: participation is strongly based on sharing the same rituals and traditions between members [12].

Therefore, for many riders, riding a motorcycle is a social experience, and that social component can significantly enhance the riding experience [2]. For example, motorcyclists passing by each other on a road may not be seen as a social event. However, even these brief and spontaneous encounters with other like-minded motorcyclists can be very valued. Indeed, sometimes motorcyclists show their appreciation by waving each other. Other forms of social interaction include riding more to promote those encounters, and engaging online [4].

However, there are also major risks associated with the use of digital tools while riding. For example, mobile phone usage during motorcycle riding constitutes a risky behavior associated with accidents and fatalities, although highly prevalent among motorcyclists [13-18]. In fact, research shows that bikers perform several operations on their mobile phones while riding, such as dialing, talking, texting, or searching for information $[13,15,17,19]$. For that reason, it is crucial that new technologies incorporate adapted and safe modes of interaction. 


\section{$3 \quad$ Research Methodology}

To address the research goals of this study, we conducted four focus group sessions, in which motorcyclists were invited to discuss ideas, needs and concerns related to different perspectives of social applications. A total of 25 participants (24 men and one woman) between 24 and 54 years-old $(\mathrm{M}=36.28, \mathrm{SD}=10.15)$ participated in these four sessions, each with 5 to 8 people. All participants owned and were regular riders of a motorcycle.

Two discussion guides were created to conduct the focus groups, and participants were randomly assigned to one of them. All groups started by exploring (1) the experience of riding a two-wheeled vehicle, and (2) the use of connected technologies. Then, two groups discussed (3) the willingness to share information and (4) the expected trade-offs of that sharing; the other two groups discussed topics not explored in the present study. Before the focus group session, participants were asked to respond to a short questionnaire about their riding routines, their preferred brands and the use of applications.

On each session, there was a moderator and a note-taker. At the beginning, participants were informed about the objectives of the focus group, and were told that there were no right or wrong answers, and that they could stop the session anytime. It was additionally explained that the session was going to be recorded and that all video and audiotapes were confidential and could only be used by researchers of the project. After that, all participants signed an informed consent. The moderator explained the session rules (e.g., one person talks at a time) and started the session following the respective guide. The sessions took between 60 and 90 minutes.

The audio data collected during the sessions was transcribed from audio to text. We then applied qualitative content analysis to the transcripts [20]. A first reading of the transcriptions and session notes allowed us to reach a general perspective on the topics addressed by participants. We then followed a coding protocol to classify transcriptions segments into categories with similar meaning. During the coding process, however, emerging categories that were not previously included in the initial protocol were also added. Therefore, the analysis was conducted following an inductive approach, by including categories that were identified in participants' speech, as well as a deductive approach that considered the coding protocol developed for this study [20]. The coding protocol was thus constantly reorganized throughout the data analysis process. Towards the end of the process, we started an aggregation effort in which each category was organized into higher-order specific dimensions, and then into more general dimensions [21].

\section{$4 \quad$ Results}

The questionnaire filled before the focus group showed that most riders used their motorcycle on both recreational and daily/commuting contexts (64\%) and only $36 \%$ of the participants reported riding for recreational purposes only. The most common brand was Honda (32\%), followed by Yamaha (24\%), and BMW (16\%). 
Eleven participants have been riding for less than 5 years, and, among those, four were new riders, riding for less than one year. Five participants reported riding for between 5 and 10 years; five between 10 and 20 years; and four for over 20 years.

Twelve participants reported to ride the motorcycle at least four times per week, five between 1 and 3 times per week and eight a maximum of once per month. Most of the participants (60\%) reported that their rides are usually short, taking on average between 15 minutes and one hour. Only $32 \%(\mathrm{n}=8)$ of the participants use a navigation system, with Google Maps being the most preferred application $(n=6)$. Most of the participants $(64 \%)$ are not members of any community or motorcycle related group.

The results of the focus-group sessions were organized around three major themes that emerged during the qualitative analysis of the data: (1) planning a trip, (2) sharing information, and (3) communicating. For each theme we report the user's current practices and their needs.

\subsection{Planning a trip}

Planning a motorcycle ride depends on several factors, such as whether it is a solo or group trip, whether it is a short or a long trip, and on the weather conditions. Current practices for trip planning seem to involve three major steps: (a) planning the route, (b) checking the motorcycle, and (c) preparing themselves (without a predefined sequence). When riding with friends the routes are chosen together, either in person or using communication applications, specially WhatsApp. To choose the route, riders rely on friends' knowledge of the surrounding areas, on web forums, and on their own searches on the map (physical or a mobile app). The most frequently used tools to plan the routes are mobile apps such as Google Maps to define interest points, restaurants, and meeting points. Some participants reported that the downside of using GPS-enabled navigation apps (as opposed to following the signs) is that sometimes travel planning is less detailed than the ideal because riders assume the app will be available at any moment:

P01: "Yes, and it is funny, because I rode a motorcycle for so many years, and in longer trips when there was no GPS or mobile phones. It was a map, and things happened, and the trip was made. It was better prepared than now, we planned better. Now we rely on the GPS and then get used to it."

Using a GPS device can interfere with safety when riding a motorcycle, because riders have to either look away from the road to look at the device, or they have to follow audio cues, and both can potentially disrupt the riding experience. Moreover, some motorcyclists prefer not to use maps at all during the trip. Also, one mountain rider preferred using a paper-map, to avoid the risk of a technological failure:

P24: “No, I don't use technologies much. (...) Or sometimes, when I go to more dangerous trails, I take a compass and a map, but they are physical because I do not want to fall, the cell phone breaks and then I end up with nothing. So, if the map tears, I can still see something ... see where I am located. But technology ... no. For what I do I don't want to depend on technology ...". 
For those willing to use technologies, the navigation system seems to be the most important one, as far as it assures safety:

P17: "More than contextualizing specific solutions, I would like something that would make my trip especially safer, that is, all those alerts about potential problems on the road... more convenient, if I want to go to a given destination, and if I want to go fast, it provides the best route; But if I want to go for leisure, it provides the most scenic route."

\subsection{Sharing information}

The second topic, willingness to share information with others, was especially important for our focus on social applications. Three major questions have emerged regarding information sharing: (1) who to share with, (2) what to share, and (3) what not to share.

Regarding whom, riders are generally available to share information with the community, so everyone can provide and receive useful information and benefit from it. Sharing information with manufacturers and brands would also be possible, but in those cases, participants expressed that they would be consider to be fair if they could also benefit from it:

P14: "If there are companies that can benefit, I think they should also give something in return, e.g. a little check-up [for the motorcycle] or something."

On the one hand, companies could benefit by collecting data on motorcycle use, and by creating closer relationships with the customers. On the other hand, users could benefit from special offers or premium services.

Regarding what type of information motorcyclists would like to share and receive from others, navigation and safety were the most common topics. Motorcyclists believe that if all road users are connected, they should all benefit from receiving alerts of proximity of other vehicles. Participants would also like to receive recommendations about the speed limit according to the type of pavement, points of interest along the route, closed roads, accidents, transit, etc. It would also be useful to have real-time weather information along the route. Concerning more specifically the roads, they could be categorized based on their scenery, and pleasure to drive (based on curves and straight roads). Also, these routes could be shared:

P05: "If it could be connected to Facebook, or something. That there were people you know and who ride motorcycles too ... yes, I don't know, if they were around, and your friend is riding a motorcycle or something, if you knew his location, things like that, even the itineraries, creating itineraries, sharing itineraries with others, or even executing those itineraries ...".

When riding in groups, the app could also allow sharing photos within the group:

P16: "It would be a sensor pack together with the application. Not for the driving experience itself but more for after [driving], to see the average consumption we had during the whole trip, the stops... But this is statistical data collection for the 
application itself, which is then good to add, one thing that is interesting to see... and there's even that part of google where you can share the photos with, if we have a group we can share if... look, we stopped at this viewpoint or something. You have some pictures, even of the motorcycle or something like that. And even share with the people who went on that trip, so everyone has access to everyone's photos.".

When riding in groups, locating everyone would also be extremely useful:

P11: "Share the location with friends, perhaps. Go meet a group of friends who also ride a motorcycle. They say, "we are here" and our GPS directly gives the indications to reach them."

Despite all the interest around information sharing, participants have also expressed some concerns and some situations where they would prefer not to share at all. They mentioned that information should be anonymous, shared content should be curated, and should not depend exclusively on the community as information providers. Ideally, part of this could be accomplished with sensors in the vehicles and in the infrastructures, so data would be less biased, and riders would not have to manually add information while driving:

P23: "The problem with Waze is that it needs to be the users to put the warning there. When we are riding a motorcycle or driving car it is not recommended to... [do it].".

Also important, information sharing should always be optional:

P05: "It could be our choice. Share or not.".

Fundamentally, participants referred two types of information they do not want to share: location and speed. Sharing the location should be done cautiously and only in certain situations. In particular, when riding alone it should only be shared with trusted people. Also, the home location should never be exposed for security reasons. In general, participants do not want to share information that can be traced back to them.

P16: "But with GPS... He left this point, went there and returned to this point, you're at home. (...) If you have GPS data, you usually can't identify who the person is, but if the starting point and the ending point are the same, it is immediately known that the person lives there. Or keeps the motorcycle there, at least."

Concerning speed, it could be shared only if it were anonymous and no negative consequences could come from that. Participants also emphasized that too much information can be distracting and especially on leisure trips, it can also interfere with the driving experience:

P16: "A lot of information when you are taking a longer trip is going to distract of what you want." 


\subsection{Communicating}

Participants often referred to various communication contexts, which mainly seem to address three types of communicating needs: (1) with people that are not riding with them (e.g., a family member at home), (2) with friends riding with them in a group, and (3) with the passenger in the motorcycle. Usually, in the first two cases conversations are short (e.g., acknowledging that she/he will be late, or signaling to stop for gas, respectively). In the last case, communication may be more continuous:

P18: "If you go in the car with your wife or your friend or whatever, you like to talk, look at the landscape. And in the motorcycle is the same. A person going there quietly for $200 \mathrm{~km}$ is a bit boring. ".

Communicating while riding can lead to dangerous situations. For example, when riding in a group, motorcyclists may try to communicate with the motorcycle light signals (e.g., using the right light signal to communicate the intent to stop) or take over everyone and shout a short message (e.g. "Stop!"). Both situations can be dangerous:

P17: "When it is a group tour, sometimes it is like, the guy who comes back needs to put gasoline and either gives light signals or have to use the turn lights because there is no easy way to communicate. Or he overtakes us all and then says "Hey, I want to stop".,"

P14: "Yes, but it ends up creating a risky situation."

P17: "Communication is not effective."

P14: "We shouldn't be doing this when traveling in a group. We should maintain our position."

Motorcyclists also referred communication difficulties while driving. On the one hand, manipulating a device is impossible or dangerous while driving.

P02: "I already did that, using applications to connect mobile phones via Bluetooth (...) for example with the passenger. For example, making a call ... there are applications for calls and a person speaks, but it ends up being distracting at the same time. So, it works, but at the same time, it distracts us.".

On the other hand, the existing communication devices, such as hands-free kits, sometimes are too expensive and when riding in groups not everyone has one:

P15: "The problem is that with some people having intercoms and others not, there is always someone who is left behind, and others that go a little bit faster, and we end up being out of sync."

One possible solution to ease communication could be to have voice interaction while riding:

P17: "I don't want to see the messages while driving because it is dangerous, but I would like to have someone reading them for me or something like that... check if I have a call waiting, something like that (...) By sound. Something like that.". 


\subsection{Other results}

This study has also identified several other relevant insights for the design of a social application. The first is the central role of safety. Even when the context is social applications, the issue of safety remains clearly amongst the top priorities. Also, most motorcyclists seem to exhibit a certain disbelief in digital technologies, at least for the riding context. While part of the problem may be associated with safety risks derived from technology use during the ride, there also seems to be some sort of disillusion with current products, particularly when compared with the thriving industry market of digital technologies for the car. Several participants have even expressed that they would just prefer not to use any digital technology at all, simply for fear that it will ruin the pleasure of riding.

\section{Conclusion}

The concept of social applications for motorcyclists can be a good fit for the socializing component of being a rider. In this study, we have explored the motorcyclists' perception about this type of digital tools. The results suggest the existence of considerable challenges involved, regarding issues such as interaction limitations, the impact on the riding experience, the very diverse set of motorcyclist profiles, or simply a general disbelief in digital tools altogether. On several occasions, participants have shown signs of resistance to technology and emphasized that information should be limited, not only for safety reasons, but also because it could interfere with the pleasure of driving.

The general conclusion is that this exploration of design opportunities for a social application for motorcyclists must be much more than a mere identification of relevant features or a simplistic attempt of recreating, in this context, the principles and concepts of other social networks. Like social networks in general, social applications for motorcyclists need to leverage the collective and socializing elements of motorcycling in a way that fits the different needs of various motorcyclists' profiles. However, their specific design should also be closely aligned with the micro-contexts of the riding experience in a way that blends smoothly with riding itself.

\section{Acknowledgements}

This work is supported by: European Structural and Investment Funds in the FEDER component, through the Operational Competitiveness and Internationalization Programme (COMPETE 2020) [Project $n^{\circ}$ 039334; Funding Reference: POCI-01-0247FEDER-039334] 


\section{References}

1. Tunnicliff, D., Watson, B., White, K.M., Lewis, I., Wishart, D.: The Social context of motorcycle riding and the key determinants influencing rider behavior: A qualitative investigation. Traffic Inj. Prev. (2011). https://doi.org/10.1080/15389588.2011.577653.

2. Jderu, G.: Motorcycles, body and risk: The motorcyclists' social career. J. Sociol. (2015). https://doi.org/10.1177/1440783312474081.

3. Maxwell, A.H.: Motorcyclists and community in post-industrial urban America. Urban anthropol. 27, 263-279 (1998).

4. Esbjörnsson, M., Juhlin, O., Östergen, M.: Motorcycling and social interaction -Design for the enjoyment of brief traffic encounters. Proc. Int. ACM Siggr. Conf. Support. Gr. Work. 85-94 (2003).

5. Coninx, P.: Riding in Big Brother's Automobile: Vehicle Technology and Consumer Privacy. Automobile Consumer Coalition, Car Help Canada (2011).

6. Spelta, C., Manzoni, V., Corti, A., Goggi, A., Savaresi, S.M.: Smartphone-Based Vehicleto-Driver/Environment Interaction System for Motorcycles. IEEE Embed. Syst. Lett. 2, 3942 (2010). https://doi.org/10.1109/LES.2010.2052019.

7. Lin, H.M., Tsai, H.M., Boban, M.: Scooter-to-X communications: Antenna placement, human body shadowing, and channel modeling. Ad Hoc Networks. 37, 87-100 (2016). https://doi.org/10.1016/j.adhoc.2015.09.006.

8. Silva, R., Iqbal, R.: Ethical Implications of Social Internet of Vehicles Systems. IEEE Internet Things J. 6, 517-531 (2019). https://doi.org/10.1109/JIOT.2018.2841969.

9. Esbjörnsson, M., Juhlin, O., Östergren, M.: Traffic encounters and Hocman: Associating motorcycle ethnography with design. Pers. Ubiquitous Comput. 8, 92-99 (2004). https://doi.org/10.1007/s00779-004-0260-4.

10. Bagozzi, R.P., Dholakia, U.M.: Antecedents and purchase consequences of customer participation in small group brand communities. Int. J. Res. Mark. 23, 45-61 (2006). https://doi.org/10.1016/j.ijresmar.2006.01.005.

11. Laroche, M., Habibi, M.R., Richard, M.-O., Sankaranarayanan, R.: The effects of social media based brand communities on brand community markers, value creation practices, brand trust and brand loyalty. Comput. Human Behav. 28, 1755-1767 (2012). https://doi.org/10.1016/j.chb.2012.04.016.

12. Madupu, V., Krishnan, B.: The relationship between online brand community participation and consciousness of kind, moral responsibility, and shared rituals and traditions. In: Advances in Consumer Research. pp. 853-854 (2008).

13. De Gruyter, C., Truong, L.T., Nguyen, H.T.T.: Who's calling? Social networks and mobile phone use among motorcyclists. Accid. Anal. Prev. 103, 143-147 (2017). https://doi.org/10.1016/j.aap.2017.04.010.

14. Widyanti, A., Pratama, G.B., Anindya, A.H., Sari, F.P., Sumali, A., Salma, S.A., Yamin, P.A.R., Soetisna, H.R.: Mobile phone use among Indonesian motorcyclists: prevalence and influencing factors. Traffic Inj. Prev. 21, 459-463 (2020). https://doi.org/10.1080/15389588.2020.1789121.

15. Phommachanh, S., Ichikawa, M., Nakahara, S., Mayxay, M., Kimura, A.: Student motorcyclists' mobile phone use while driving in Vientiane, Laos. Int. J. Inj. Contr. Saf. Promot. 24, 245-250 (2017). https://doi.org/10.1080/17457300.2016.1166141.

16. Pérez-Núñez, R., Hidalgo-Solórzano, E., Vera-López, J.D., Lunnen, J.C., Chandran, A., Híjar, M., Hyder, A.A.: The Prevalence of Mobile Phone Use Among Motorcyclists in Three Mexican Cities. Traffic Inj. Prev. 15, 148-150 (2014). https://doi.org/10.1080/15389588.2013.802776. 
17. Truong, L.T., De Gruyter, C., Nguyen, H.T.T.: Calling, texting, and searching for information while riding a motorcycle: A study of university students in Vietnam. Traffic Inj. Prev. 18, 593-598 (2017). https://doi.org/10.1080/15389588.2017.1283490.

18. Truong, L.T., Nguyen, H.T.T., De Gruyter, C.: Correlations between mobile phone use and other risky behaviours while riding a motorcycle. Accid. Anal. Prev. 118, 125-130 (2018). https://doi.org/10.1016/j.aap.2018.06.015.

19. Truong, L.T., Nguyen, H.T.T., De Gruyter, C.: Mobile phone use among motorcyclists and electric bike riders: A case study of Hanoi, Vietnam. Accid. Anal. Prev. 91, 208-215 (2016). https://doi.org/10.1016/j.aap.2016.03.007.

20. Schilling, J.: On the Pragmatics of Qualitative Assessment. Eur. J. Psychol. Assess. 22, 28 37 (2006). https://doi.org/10.1027/1015-5759.22.1.28.

21. Vaismoradi, M., Turunen, H., Bondas, T.: Content analysis and thematic analysis: Implications for conducting a qualitative descriptive study. Nurs. Health Sci. 15, 398-405 (2013). https://doi.org/10.1111/nhs.12048. 